\title{
Fabrication and Characterization of Patterned Single-Crystal Silicon
}

\section{Nanolines}

Bin Li, Min K. Kang, Kuan Lu, Rui Huang, Paul S. Ho, Richard A. Allen and Michael W.

Cresswell

1. As an experimental evidence of the nearly atomically flat sidewalls of the SiNLs fabricated in the present study, we compare the nanolines produced by anisotropic wet etching and those by reactive ion etching (Figure S1). Clearly, the present fabrication process with anisotropic wet etching yields much smoother sidewalls.
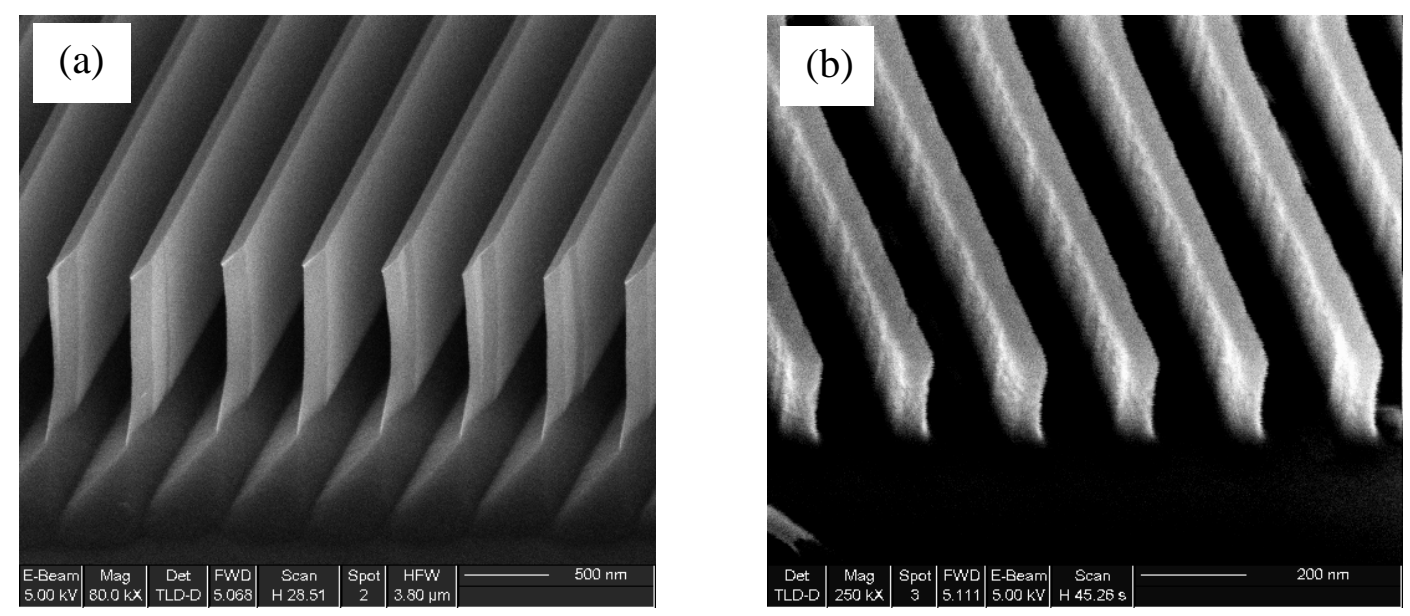

Figure S1. Cross-sectional SEM images of $65 \mathrm{~nm}$ silicon nanolines: (a) for lines fabricated by pattern transfer with anisotropic wet etching, and (b) for lines by $\mathrm{CF}_{4}+\mathrm{O}_{2}$ plasma etching. 
2. Figure S2 shows the confined buckle deformation of the SiNLs after the displacement burst, obtained from a finite element simulation. The stress distribution in the SiNLs is exhibited as contours.

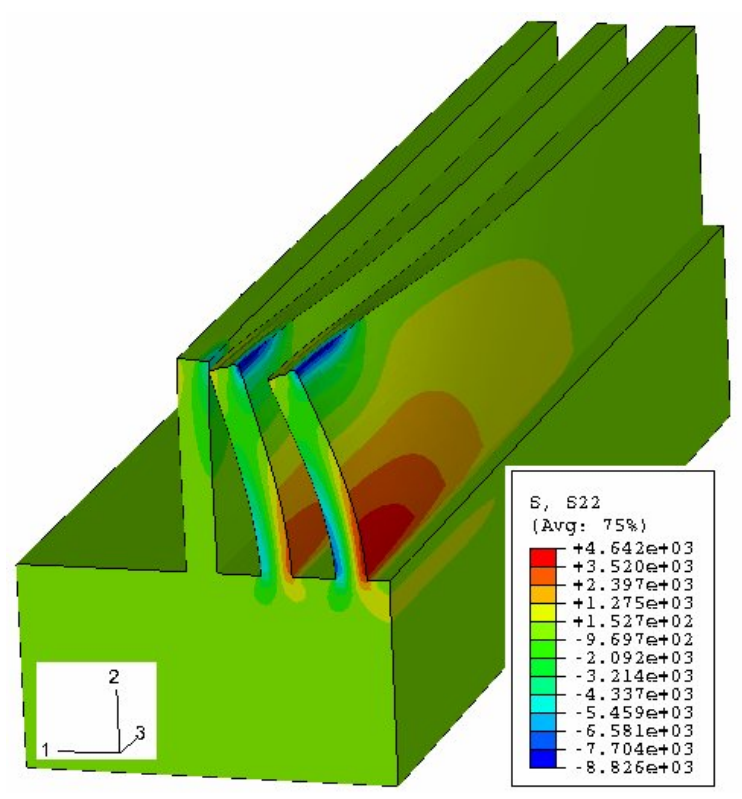

Figure S2. Deformation of SiNLs and stress distribution obtained from a finite element model. By symmetry, only one quarter of the nanolines structures is shown. Both the deformation and stress are confined locally under the indenter tip, within a distance two to three times the height of the SiNLs. 